\title{
Begleitung einer Ausschaffung durch Ärzte: ethische Aspekte
}

Jörg Nef

Korrespondenz:

Dr. med. Jörg Nef

Obere Weidegg 4

CH-9230 Flawil
Im letzten Jahr wurde in der Schweizerischen Ärztezeitung fast endlos über ethische Aspekte im Zusammenhang mit der Zwangsernährung von Gefangenen im Hungerstreik diskutiert. Über ärztliche Ethik im Zusammenhang mit der von den Behörden neu eingeführten ärztlichen Begleitung bei Zwangsausschaffungen von zurückgewiesenen Asylbewerbern wurde kein Wort verloren.

Nun ist in der Schweizerischen Ärztezeitung Nr. 10 vom 9. März 2011 ein hervorragender Artikel der Herren Viktor Györffy, Rechtsanwalt in Zürich, und Michel Romanens, Kardiologe aus Olten, erschienen. Er hat den Titel «Tod durch Ausschaffung: Bemerkung zum Level IV» und die beiden Untertitel «Begleitung einer Ausschaffung durch Ärzte: juristische Aspekte» respektive «... medizinische Aspekte». Den Titel meiner Ausführungen habe ich in Analogie zu diesen beiden genannten Untertiteln gewählt, quasi als dritten Teil des Artikels der Herren Györffy und Romanens. Sie mögen mir das nicht übel nehmen.

Bei der Zwangsausschaffung eines abgewiesenen Asylbewerbers wird staatliche Gewalt angewendet, darüber besteht kein Zweifel. Im März 2010 starb ein Asylbewerber bei der Zwangsausschaffung, noch bevor er - an einen Transportstuhl gefesselt - das Flugzeug erreichte (vgl. die Abbildung im oben genannten Artikel auf Seite 384). Nach einer mehrmonatigen Pause wurde die Praxis der Zwangsausschaffung wieder aufgenommen, neu mit ärztlicher Begleitung.

Die medizinische Überwachung staatlicher Gewaltanwendung ist keine ärztliche Aufgabe. Der Arzt muss körperliche Gewaltanwendung an einem Menschen verhindern, nicht begleiten. Jetzt soll aber ein Mitglied unserer Zunft dafür sorgen, dass die Staatsgewalt medizinisch korrekt abläuft, und er soll allfällige medizinische Komplikationen verhindern oder beheben. Der erwähnte Asylbewerber ist nicht in erster Linie gestorben, weil kein Begleitarzt anwesend war, sondern weil körperliche und psychische Gewalt gegen ihn eingesetzt wurden, vorbestehende Herzerkrankung hin oder her. Trotzdem will nun der Staat einen Arzt verpflichten, damit während der Zwangsausschaffung keine medizinischen Fehler gemacht werden, der Arzt dafür die Verantwortung übernimmt und damit den Staat bezüglich gesundheitlicher Komplikationen oder sogar Todeseintritt entlastet. Das ist unzulässig. Erstens ist körperliche Gewaltanwendung an sich aus medizinischer und ärztlicher Sicht nicht korrekt, und zweitens müssen der Staat und seine entsprechenden Funktionäre allfällige medizinische Komplikationen einer Zwangsausschaffung vollum- fänglich selbst verantworten. Kein Arzt darf ihm diese Verantwortung abnehmen, sonst verkommt er zum Handlanger des Staates bei dessen körperlicher Gewaltanwendung gegenüber wehrlosen Menschen.

In den USA ist bei der Vollstreckung der Todesurteile ein Arzt anwesend, damit bei der Tötung kein medizinischer Fehler gemacht wird. Die Exekution muss medizinisch sauber über die Bühne gehen. Die Auslöschung eines Lebens ist aber an sich der medizinische Kapitalfehler, die medizinische Katastrophe. Kein Arzt darf sich dazu hergeben, eine Tötung medizinisch zu assistieren.

Ein Schweizer Sprayer wurde letztes Jahr in China zu drei Stockschlägen verurteilt. Ein Arzt sollte bei der Bestrafung dabei sein und dafür sorgen, dass die Stockschläge medizinisch korrekt plaziert werden. Das ist keine ärztliche Aufgabe, das ist pervers.

Das dunkelste Kapitel ärztlicher Assistenz bei körperlicher Gewaltanwendung durch den Staat hat sich im Verlauf der ganzen Menschheitsgeschichte in Nazideutschland abgespielt. Tausende von Ärzten haben im 12 Jahre dauernden «Tausendjährigen Reich» dem Staat geholfen, Juden, politisch Andersdenkende, Zigeuner, Behinderte und Geisteskranke umzubringen, um Regimegegner auszuschalten und eine reine deutsche Rasse zu züchten.

Die Unterschiede zwischen den erwähnten drei Beispielen und der ärztlichen Begleitung von Zwangsausschaffungen sind gradueller, nicht prinzipieller Natur. Es handelt sich in allen Fällen um ärztliche Assistenz bei körperlicher und geistiger Gewaltanwendung des Staates gegenüber Wehrlosen. Der bei einer Zwangsausschaffung anwesende Arzt soll es dem Staat ermöglichen, Gewalt anzuwenden ohne gesundheitliches Risiko für den Betroffenen oder gar Todesfolge, und macht sich damit zum Komplizen der Staatsgewalt.

Zum Abschluss nochmals zurück zum oben genannten Artikel: Der Schlusssatz lautet dort: «Die Teilnahme von Ärztinnen und Ärzten an solchen Prozeduren muss abgelehnt werden.» Dies gilt auch in ethischer Hinsicht. Im Fall der beschriebenen Zwangsausschaffung des K. A. hatte der Gefängnisarzt den Gesundheitszustand falsch eingeschätzt und vor der Abreise des Asylbewerbers die Fesselung an den Transportstuhl und die Knebelung als medizinisch unbedenklich eingestuft. Die Abschiebung der Verantwortung auf den Arzt hat bestens geklappt. Ausschaffungsbehörde und Polizei gingen straffrei aus, der Arzt wurde wegen fahrlässiger Tötung (!) verurteilt. Er war Teil der Ausschaffungsorgane und hat damit teilgenommen an der staatlichen Gewaltanwendung, er war Komplize ... 